\title{
Erratum to: Estimating Energy Requirements
}

Jacalyn J. Robert-McComb, Elvis Álvarez Carnero, and Eduardo Iglesias-Gutiérrez

\section{Erratum to:}

\section{Chapter 27 in: J.J. Robert-McComb et al. (eds.),}

The Active Female: Health Issues Throughout the Lifespan, https://doi.org/10.1007/978-1-4614-8884-2_27

The print and online versions of the book were incorrectly published with Mathematical errors in Chapter 27, pages 424-426. The errors have now been updated with these changes. The correct equations are given below

\section{Chapter 27, Page 424}

\subsubsection{Estimating TEE: A Case Study}

Alice is a 37- year-old woman, weight $64 \mathrm{~kg}$, should be

Alice is a 37- year-old woman, weight $75 \mathrm{~kg}$

\section{Page 425}

First Step.

$\mathrm{FFM}=75-(0.12 \times 75)=75-(9)=64 \mathrm{~kg}$.

Should be

$\mathrm{FFM}=75-(0.12 \times 75)=75-9=66 \mathrm{~kg}$.

$\mathrm{RMR}=24.6 \times(64 \mathrm{~kg})+175=1,749 \mathrm{kcal} /$ day.

$\operatorname{RMR}(\mathrm{kcal} / \mathrm{h})=1,749 / 24=72.9 \mathrm{kcal} / \mathrm{h}$

Should be

$\mathrm{RMR}=24.6 \times(66 \mathrm{~kg})+175=1,798.6 \mathrm{kcal} /$ day.

$\operatorname{RMR}(\mathrm{kcal} / \mathrm{h})=1,798.6 / 24=74.94 \mathrm{kcal} / \mathrm{h}$

The updated online version of this chapter can be found at https://doi.org/10.1007/978-1-4614-8884-2_27 


\section{Second step.}

3. EE of sleeping $=0.9 \times 72.9 \mathrm{kcal} / \mathrm{h}=65.6 \mathrm{kcal} / \mathrm{h}$.

Should be:

3. EE of sleeping $=0.9 \times 74.94 \mathrm{kcal} / \mathrm{h}=67.4 \mathrm{kcal} / \mathrm{h}$.

Total EE of sleeping $=65.6 \times 5=328 \mathrm{kcal} /$ day .

Should be

Total EE of sleeping $=67.4 \times 5=337 \mathrm{kcal} / \mathrm{day}$.

\section{Page 426}

\section{Calculations:}

3. EE during sedentary leisure $=1 \mathrm{~h} \times 2.0$

METs $=3.9 \mathrm{kcal} / \mathrm{kg} \times 75 \mathrm{~kg}=150 \mathrm{kcal} /$ day.

Should be

3. EE during sedentary leisure $=1 \mathrm{~h} \times 2.0$

$\mathrm{METs}=2 \mathrm{kcal} / \mathrm{kg} \times 75 \mathrm{~kg}=150 \mathrm{kcal} /$ day .

\section{Sixth step.}

$T E E=2759 \mathrm{kcal} / \mathrm{day}$

$P A L=2759(\mathrm{kcal} /$ day $) / 1749(\mathrm{kcal} /$ day $)=1.5$

Should be

TEE $=2759 \mathrm{kcal} /$ day

$P A L=2759(\mathrm{kcal} /$ day $) / 1798.6(\mathrm{kcal} /$ day $)=1.5$

\section{Page 426, line 15-20}

\subsubsection{NEAT Estimation}

All together NEAT physical activities lasted $13 \mathrm{~h}$, and RMR was $72.9 \mathrm{kcal} / \mathrm{h}$. So we need to multiply $13 \mathrm{~h} \times 72.9 \mathrm{kcal} / \mathrm{h}$ in order to calculate kcal that were expended for resting during NEAT activities, which were $948 \mathrm{kcal}$. So final net NEAT was 1,483 during a day.

Should be

All together NEAT physical activities lasted $13 \mathrm{~h}$, and RMR was $74.94 \mathrm{kcal} / \mathrm{h}$. So we need to multiply $13 \mathrm{~h} \times 74.94 \mathrm{kcal} / \mathrm{h}$ in order to calculate $\mathrm{kcal}$ that were expended for resting during NEAT activities, which were $974 \mathrm{kcal}$. So final net NEAT was 1,457 kcal during a day.

Final NEAT Results 2,431, kcal/day -948 kcal/ day $=1,483 \mathrm{kcal} /$ day

Should be

Final NEAT Results 2,431, kcal/day-974 kcal/ day $=1,457 \mathrm{kcal} /$ day 